Turk. J. Math. Comput. Sci.

13(2)(2021) 261-269

(C) MatDer

DOI : $10.47000 /$ tjmcs.858489

\title{
$(k, m)$-type Slant Helices According to Parallel Transport Frame in Euclidean 4-Space
}

\author{
Fatma Bulut $^{1}$ (D), Feyzi TARTiK ${ }^{1, *}$ (D) \\ ${ }^{1}$ Department of Mathematics, Faculty of Arts and Sciences, Bitlis Eren University, 13000, Bitlis, Turkey.
}

Received: 11-01-2021 • Accepted: 31-07-2021

\begin{abstract}
Aвstract. In this work, we describe a Frenet frame in 4-dimensional Euclidean space and call this frame as parallel transport frame (PTF). PTF is an alternative approach to defining a moving frame. This frame is obtained by rotating the tangent vector and the first binormal vector of a unit speed curve by an euler angle and then we give curvature functions according to PTF of the curve. Also, we introduce $(k, m)$-type slant helices according to PTF in Euclidean 4-Space. Additionally, we obtain the characterization of slant helices according to this frame in $\mathbb{E}^{4}$ and give an example of our main result.
\end{abstract}

2010 AMS Classification: $14 \mathrm{H} 50,53 \mathrm{~A} 04$

Keywords: Slant helix, curvatures, Euclidean 4-space, parallel transport frame.

\section{INTRODUCTION}

Some special curves and surfaces are of great importance in differential geometry. Frenet equations and curvatures of the curve play a key role in studies on curves. Frenet equations are used in the construction of many curve theories, and offer interesting results. One of the most important of these curves is the helix curve and the characterizations on the curvature and torsion play an important role to define special curve types such as so-called helices. Helices are defined as curves whose tangent vector makes a constant angle with a fixed direction. The curve is a $(k, m)$-type slant helix, then there exists a non-zero constant vector field, and with this constant vector field, the vector fields which have the same index of a parallel transport frame make a constant angle (as in the Euclidean space $\mathbb{E}^{4}$ ). Recently, many research papers related to this concept. The slant helix concept in Euclidean 3-space is defined by Izumiya and Takeuchi [7]. M.A. Soliman, N. H.Abdel-All, R. A. Hussien and T. Youssef studied [8] evolution of space curves using type-3 Bishop frame. S. Yilmaz and M. Turgut $[10,11]$ introduced a new version of Bishop frame and an application to spherical images. M.Y. Yilmaz and M. Bektaş defined $(k, m)$-type slant helices in 4-dimensional Euclidean space and null curves in Minkowski 4-space [1,9]. In addition, F. Bulut and M. Bektaş [3] obtained special helices on Equiform differential geometry of spacelike curves in Minkowski space-time. On the other hand, the Bishop frame was introduced by R. L. Bishop in 1975 utilizing parallel vector fields [2].

In the working, the parallel transport frame (PTF) are studied. The aim of this paper is to introduce slant helix in Euclidean 4-Space according to PTF. In particular, the definition of PTF created with a $t$ parameter is granted. Subsequently, important characterizations are given for PTF to be $(k, m)$-type slant helix in 4-dimensional Euclidean space. Also, it is given an example for the curve in $\mathbb{E}^{4}$.

*Corresponding Author

Email addresses: fbulut@ beu.edu.tr (F. Bulut), ftartik69@gmail.com (F. Tartık) 


\section{Geometric Preliminaries}

In this section we present basic tools for the space curves in $\mathbb{E}^{4}$. A detailed information can be found in [5]. Let $\gamma: I \subset R \rightarrow \mathbb{E}^{4}$ be an arbitrary curve in Euclidean space $\mathbb{E}^{4}$. Let $\mathbb{E}^{4}=\left\{\left(a_{1}, a_{2}, a_{3}, a_{4}\right) \mid a_{1}, a_{2}, a_{3}, a_{4} \in \mathbb{R}\right\}$ be a 4-dimensional vector space. The standard scalar product in $\mathbb{E}^{4}$ given by

$$
\langle a, b\rangle=\sum_{k=1}^{4} a_{k} b_{k} .
$$

Then the curve $\gamma$ is said to be of unit speed (or parametrized by arclength) if it satisfies $\langle\dot{\gamma}(t), \dot{\gamma}(t)\rangle=1$ for any $t \in I$. The norm of a vector $a \in \mathbb{E}^{4}$ is defined by $\|a\|=\sqrt{|\langle a, a\rangle|}$.

Let $\left\{\mathbf{T}, \mathbf{N}, \mathbf{B}_{\mathbf{1}}, \mathbf{B}_{\mathbf{2}}\right\}$ be the moving frame along the unit speed curve $\gamma$; where $\mathbf{T}(t), \mathbf{N}(t), \mathbf{B}_{\mathbf{1}}(t)$ and $\mathbf{B}_{\mathbf{2}}(t)$ denote, the tangent, the principal normal, first and second binormal vector fields of the curve $\gamma$, respectively. Then the following Frenet formulas is given in [11]

$$
\left[\begin{array}{c}
\mathbf{T} \\
\mathbf{N} \\
\mathbf{B}_{1} \\
\mathbf{B}_{2}
\end{array}\right]^{\prime}=\left[\begin{array}{cccc}
0 & \kappa_{1} & 0 & 0 \\
-\kappa_{1} & 0 & \kappa_{2} & 0 \\
0 & -\kappa_{2} & 0 & \kappa_{3} \\
0 & 0 & -\kappa_{3} & 0
\end{array}\right]\left[\begin{array}{c}
\mathbf{T} \\
\mathbf{N} \\
\mathbf{B}_{1} \\
\mathbf{B}_{2}
\end{array}\right]
$$

where $\kappa_{1}, \kappa_{2}$ and $\kappa_{3}$ denote the first, the second and the third curvature functions according to of $\gamma$, respectively. Here, since $\left\{\mathbf{T}, \mathbf{N}, \mathbf{B}_{\mathbf{1}}, \mathbf{B}_{\mathbf{2}}\right\}$ is an orthonormal frame, then we can write

$$
\begin{aligned}
\langle\mathbf{T}, \mathbf{T}\rangle & =\langle\mathbf{N}, \mathbf{N}\rangle=\left\langle\mathbf{B}_{\mathbf{1}}, \mathbf{B}_{1}\right\rangle=\left\langle\mathbf{B}_{2}, \mathbf{B}_{2}\right\rangle=1, \\
\langle\mathbf{T}, \mathbf{N}\rangle=\left\langle\mathbf{T}, \mathbf{B}_{1}\right\rangle & =\left\langle\mathbf{T}, \mathbf{B}_{2}\right\rangle=\left\langle\mathbf{N}, \mathbf{B}_{1}\right\rangle=\left\langle\mathbf{N}, \mathbf{B}_{2}\right\rangle=\left\langle\mathbf{B}_{\mathbf{1}}, \mathbf{B}_{2}\right\rangle=0 .
\end{aligned}
$$

Now, we rotate this frame in $\mathbb{E}^{4}$ arbitrarily. So, when we rotate $\left\{\mathbf{T}, \mathbf{N}, \mathbf{B}_{\mathbf{1}}, \mathbf{B}_{\mathbf{2}}\right\}$ vectors, we get $\left\{\mathbf{T}^{*}, \mathbf{N}^{*}, \mathbf{B}_{\mathbf{1}}{ }^{*}, \mathbf{B}_{\mathbf{2}}{ }^{*}\right\}$ vectors. If we sort the $\left\{\mathbf{T}, \mathbf{N}, \mathbf{B}_{\mathbf{1}}, \mathbf{B}_{\mathbf{2}}\right\}$ vectors as $\left\{V_{1}, V_{2}, V_{3}, V_{4}\right\}$ vectors in order, then $\left\{\mathbf{T}^{*}, \mathbf{N}^{*}, \mathbf{B}_{\mathbf{1}}{ }^{*}, \mathbf{B}_{\mathbf{2}}{ }^{*}\right\}$ vectors will be $\left\{V_{1}^{*}, V_{2}^{*}, V_{3}^{*}, V_{4}^{*}\right\}$. The Euler angles are introduced by Leonhard Euler to describe the orientation of a rigid body with respect to a fixed coordinate system [6]. Alternative forms can represent the orientation of a mobile frame of reference in physics or the orientation of a general basis in 3-dimensional linear algebra. If $\theta$ is an Euler angle, using we using $\theta_{i j}$ Euler angles between the vector $V_{i}$ and the vector $V_{j}^{*}, 1 \leq i, j \leq 4$, then the rotational transform is given by an arbitrary rotation matrix below:

$$
\left[\begin{array}{c}
V_{1}^{*} \\
V_{2}^{*} \\
V_{3}^{*} \\
V_{4}^{*}
\end{array}\right]=\left[\begin{array}{llll}
\cos \theta_{11} & \cos \theta_{21} & \cos \theta_{31} & \cos \theta_{41} \\
\cos \theta_{12} & \cos \theta_{22} & \cos \theta_{32} & \cos \theta_{42} \\
\cos \theta_{13} & \cos \theta_{23} & \cos \theta_{33} & \cos \theta_{43} \\
\cos \theta_{14} & \cos \theta_{24} & \cos \theta_{34} & \cos \theta_{44}
\end{array}\right]\left[\begin{array}{c}
V_{1} \\
V_{2} \\
V_{3} \\
V_{4}
\end{array}\right] .
$$

Here, if we leave the $\mathbf{N}$ and $\mathbf{B}_{\mathbf{2}}$ vectors motionless, we rotate only the vectors $\mathbf{T}$ and $\mathbf{B}_{\mathbf{1}}$ in the $\left\{\mathbf{T}, \mathbf{B}_{\mathbf{1}}\right\}$ plane by an angle $\theta$. Then, we can find

$$
\left[\begin{array}{c}
V_{1}^{*} \\
V_{2}^{*} \\
V_{3}^{*} \\
V_{4}^{*}
\end{array}\right]=\left[\begin{array}{cccc}
\cos \theta & \cos \left(\frac{\pi}{2}\right) & \cos \left(\frac{\pi}{2}-\theta\right) & \cos \left(\frac{\pi}{2}\right) \\
\cos \left(\frac{\pi}{2}\right) & \cos (0) & \cos \left(\frac{\pi}{2}\right) & \cos \left(\frac{\pi}{2}\right) \\
\cos \left(\frac{\pi}{2}+\theta\right) & \cos \left(\frac{\pi}{2}\right) & \cos \theta & \cos \left(\frac{\pi}{2}\right) \\
\cos \left(\frac{\pi}{2}\right) & \cos \left(\frac{\pi}{2}\right) & \cos \left(\frac{\pi}{2}\right) & \cos (0)
\end{array}\right]\left[\begin{array}{c}
V_{1} \\
V_{2} \\
V_{3} \\
V_{4}
\end{array}\right]
$$

PTF of a curve and the relations between the frame and Frenet frame of the curve in 4-dimensional Euclidean space by using the Euler angles is given in [4]. In this section, we proved with this similar thinking the following theorem.

Theorem 2.1. Let $\left\{\mathbf{T}(t), \mathbf{N}(t), \mathbf{B}_{1}(t), \mathbf{B}_{2}(t)\right\}$ be a Frenet frame along a unit speed curve $\gamma: I \subset \mathbb{R} \rightarrow \mathbb{E}^{4}$ and $\left\{\overline{\mathbf{T}}(t), \overline{\mathbf{N}}(t), \overline{\mathbf{B}_{1}}(t), \overline{\mathbf{B}_{2}}(t)\right\}$ denotes the PTF of the curve $\gamma$. The relation can be expressed as

$$
\begin{aligned}
& \overline{\mathbf{T}}(t)=\mathbf{T}(t) \cos \theta(t)+\mathbf{B}_{\mathbf{1}}(t) \sin \theta(t) \\
& \overline{\mathbf{N}}(t)=\mathbf{N}(t) \\
& \overline{\mathbf{B}_{1}}(t)=-\mathbf{T}(t) \sin \theta(t)+\mathbf{B}_{\mathbf{1}}(t) \cos \theta(t) \\
& \overline{\mathbf{B}_{2}}(t)=\mathbf{B}_{\mathbf{2}}(t) .
\end{aligned}
$$


The alternative parallel transport frame equations are

$$
\left[\begin{array}{c}
\overline{\mathbf{T}} \\
\overline{\mathbf{N}} \\
\overline{\mathbf{B}_{\mathbf{1}}} \\
\overline{\mathbf{B}_{2}}
\end{array}\right]^{\prime}=\left[\begin{array}{cccc}
0 & \overline{\kappa_{1}} & 0 & \tan \theta \overline{\kappa_{3}} \\
-\overline{\kappa_{1}} & 0 & \overline{\kappa_{2}} & 0 \\
0 & -\overline{\kappa_{2}} & 0 & \overline{\kappa_{3}} \\
-\tan \theta \overline{\kappa_{3}} & 0 & -\overline{\kappa_{3}} & 0
\end{array}\right]\left[\begin{array}{c}
\overline{\mathbf{T}} \\
\overline{\mathbf{N}} \\
\overline{\mathbf{B}_{\mathbf{1}}} \\
\overline{\mathbf{B}_{\mathbf{2}}}
\end{array}\right],
$$

where $\overline{\kappa_{1}}, \overline{\kappa_{2}}, \overline{\kappa_{3}}$ are curvature functions according to PTF of the curve $\gamma$ and their expression as follows

$$
\begin{aligned}
& \overline{\kappa_{1}}(t)=\kappa_{1}(t) \cos \theta(t)-\kappa_{2}(t) \sin \theta(t) \\
& \overline{\kappa_{2}}(t)=\kappa_{2}(t) \cos \theta(t)+\kappa_{1}(t) \sin \theta(t) \\
& \overline{\kappa_{3}}(t)=\kappa_{3}(t) \cos \theta(t)
\end{aligned}
$$

where $\theta(t)=$ constant, $\theta(t) \in\left[-\frac{\pi}{2}, \frac{\pi}{2}\right]$.

Proof. Let $\left\{\mathbf{T}(t), \mathbf{N}(t), \mathbf{B}_{1}(t), \mathbf{B}_{\mathbf{2}}(t)\right\}$ be a Frenet frame and $\left\{\overline{\mathbf{T}}(t), \overline{\mathbf{N}}(t), \overline{\mathbf{B}_{1}}(t), \overline{\mathbf{B}_{\mathbf{2}}}(t)\right\}$ denotes the PTF along a unit speed curve $\gamma$ in $\mathbb{E}^{4}$. The relation between Frenet frame and PTF as follows

$$
\left[\begin{array}{c}
\overline{\mathbf{T}} \\
\overline{\mathbf{N}} \\
\overline{\mathbf{B}_{\mathbf{1}}} \\
\overline{\mathbf{B}_{\mathbf{2}}}
\end{array}\right]=\left[\begin{array}{cccc}
\cos \theta(t) & 0 & \sin \theta(t) & 0 \\
0 & 1 & 0 & 0 \\
-\sin \theta(t) & 0 & \cos \theta(t) & 0 \\
0 & 0 & 0 & 1
\end{array}\right]\left[\begin{array}{c}
\mathbf{T} \\
\mathbf{N} \\
\mathbf{B}_{\mathbf{1}} \\
\mathbf{B}_{\mathbf{2}}
\end{array}\right] .
$$

Since the matrix in the middle is an orthogonal matrix, the inverse exists and from inverse matrix, we find

$$
\begin{aligned}
& \mathbf{T}=\overline{\mathbf{T}} \cos \theta(t)-\overline{\mathbf{B}_{1}} \sin \theta(t) \\
& \mathbf{N}=\overline{\mathbf{N}} \\
& \mathbf{B}_{1}=\overline{\mathbf{T}} \sin \theta(t)+\overline{\mathbf{B}_{1}} \cos \theta(t) \\
& \mathbf{B}_{2}=\overline{\mathbf{B}_{2}} .
\end{aligned}
$$

Differentiating (2.2) with respect to $t$, we get

$$
\begin{aligned}
& \overline{\mathbf{T}}^{\prime}=\mathbf{T}^{\prime} \cos \theta(t)-\mathbf{T} \sin \theta(t) \theta^{\prime}(t)+\mathbf{B}_{\mathbf{1}}{ }^{\prime} \sin \theta(t)+\mathbf{B}_{\mathbf{1}} \cos \theta(t) \theta^{\prime}(t) \\
& \overline{\mathbf{N}}^{\prime}=\mathbf{N}^{\prime} \\
& \overline{\mathbf{B}}_{\mathbf{1}}{ }^{\prime}=-\mathbf{T}^{\prime} \sin \theta(t)-\mathbf{T} \cos \theta(t) \theta^{\prime}(t)+\mathbf{B}_{\mathbf{1}}{ }^{\prime} \cos \theta(t)-\mathbf{B}_{\mathbf{1}} \sin \theta(t) \theta^{\prime}(t) \\
& \overline{\mathbf{B}}_{\mathbf{2}}{ }^{\prime}=\mathbf{B}_{\mathbf{2}}{ }^{\prime},
\end{aligned}
$$

where substituting the equation (2.1) instead of $\mathbf{T}^{\prime}(t), \mathbf{N}^{\prime}(t), \mathbf{B}_{\mathbf{1}}{ }^{\prime}(t), \mathbf{B}_{\mathbf{2}}{ }^{\prime}(t)$, we get

$$
\begin{aligned}
& \overline{\mathbf{T}}^{\prime}(t)=\kappa_{1} \mathbf{N} \cos \theta-\mathbf{T}(t) \sin \theta \theta^{\prime}+\left(-\kappa_{2} \mathbf{N}+\kappa_{3} \mathbf{B}_{2}\right) \sin \theta+\mathbf{B}_{\mathbf{1}}(t) \cos \theta \theta^{\prime} \\
& \overline{\mathbf{N}}^{\prime}(t)=\mathbf{N}^{\prime}=-\kappa_{1} \mathbf{T}+\kappa_{2} \mathbf{B}_{\mathbf{1}} \\
& \overline{\mathbf{B}_{\mathbf{1}}}{ }^{\prime}(t)=-\kappa_{1} \mathbf{N} \sin \theta-\mathbf{T}(t) \cos \theta \theta^{\prime}+\left(-\kappa_{2} \mathbf{N}+\kappa_{3} \mathbf{B}_{2}\right) \cos \theta-\mathbf{B}_{\mathbf{1}}(t) \sin \theta \theta^{\prime} \\
& \overline{\mathbf{B}} \mathbf{2}^{\prime}(t)=\mathbf{B}_{\mathbf{2}}{ }^{\prime}=-\kappa_{3} \mathbf{B}_{\mathbf{1}}
\end{aligned}
$$

and (2.3) results are written instead of $\mathbf{T}, \mathbf{N}, \mathbf{B}_{1}, \mathbf{B}_{\mathbf{2}}$, we have

$$
\begin{aligned}
& \overline{\mathbf{T}}^{\prime}=\left(\kappa_{1} \cos \theta(t)-\kappa_{2} \sin \theta(t)\right) \overline{\mathbf{N}}+\theta^{\prime}(t) \overline{\mathbf{B}_{\mathbf{1}}}+\kappa_{3} \sin \theta(t) \overline{\mathbf{B}_{\mathbf{2}}} \\
& \overline{\mathbf{N}}^{\prime}=\left(\kappa_{2} \sin \theta(t)-\kappa_{1} \cos \theta(t)\right) \overline{\mathbf{T}}+\left(\kappa_{2} \cos \theta(t)+\kappa_{1} \sin \theta(t)\right) \overline{\mathbf{B}_{\mathbf{1}}} \\
& {\overline{\mathbf{B}_{\mathbf{1}}}}^{\prime}=-\overline{\mathbf{T}} \theta^{\prime}(t)-\left(\kappa_{1} \sin \theta(t)+\kappa_{2} \cos \theta(t)\right) \overline{\mathbf{N}}+\kappa_{3} \cos \theta(t) \overline{\mathbf{B}_{\mathbf{2}}} \\
& {\overline{\mathbf{B}_{\mathbf{2}}}}^{\prime}=-\kappa_{3} \sin \theta(t) \overline{\mathbf{T}}-\kappa_{3} \cos \theta(t) \overline{\mathbf{B}_{\mathbf{1}}},
\end{aligned}
$$

where $\theta(t)=$ constant, $\theta^{\prime}(t)=0$ and $\theta(t) \in\left[-\frac{\pi}{2}, \frac{\pi}{2}\right]$. Then, we define this frame as PTF [10]. So, the PTF formulas are given by

$$
\left[\begin{array}{c}
\overline{\mathbf{T}} \\
\overline{\mathbf{N}} \\
\overline{\mathbf{B}_{\mathbf{1}}} \\
\overline{\mathbf{B}_{2}}
\end{array}\right]^{\prime}=\left[\begin{array}{cccc}
0 & \overline{\kappa_{1}} & 0 & \tan \theta \overline{\kappa_{3}} \\
-\overline{\kappa_{1}} & 0 & \overline{\kappa_{2}} & 0 \\
0 & -\overline{\kappa_{2}} & 0 & \overline{\kappa_{3}} \\
-\tan \theta \overline{\kappa_{3}} & 0 & -\overline{\kappa_{3}} & 0
\end{array}\right]\left[\begin{array}{c}
\overline{\mathbf{T}} \\
\overline{\mathbf{N}} \\
\overline{\mathbf{B}_{\mathbf{1}}} \\
\overline{\mathbf{B}_{2}}
\end{array}\right],
$$


where $\theta(t)=$ constant, $\theta(t) \in\left[-\frac{\pi}{2}, \frac{\pi}{2}\right]$ and curvatures are defined by

$$
\begin{aligned}
& \overline{\kappa_{1}}=\kappa_{1} \cos \theta-\kappa_{2} \sin \theta \\
& \overline{\kappa_{2}}=\kappa_{2} \cos \theta+\kappa_{1} \sin \theta \\
& \overline{\kappa_{3}}=\kappa_{3} \cos \theta .
\end{aligned}
$$

The proof is completed.

\section{3. $(k, m)$-Type Slant Helices}

In this section, we define $(k, m)$-type slant helices for PTF in $\mathbb{E}^{4}$ such as [9].

Definition 3.1 ( [9]). Let $\gamma: I \subset \mathbb{R} \rightarrow \mathbb{E}^{4}$ be a regular unit speed curve in $\mathbb{E}^{4}$ with PTF $\left\{V_{1}, V_{2}, V_{3}, V_{4}\right\}$. We call $\gamma$ is a $(k, m)$-type slant helix if there exists a non-zero constant vector field $\mathbf{U} \in \mathbb{E}^{4}$ satisfies $\left\langle V_{k}, \mathbf{U}\right\rangle=a$ and $\left\langle V_{m}, \mathbf{U}\right\rangle=b(a, b$ are constants) for $1 \leq k, m \leq 4, k \neq m$. The constant vector $\mathbf{U}$ is on axis of $(k, m)$-type slant helix [1,9]. We decompose $\mathbf{U}$ with respect to $\operatorname{PTF}\left\{\overline{\mathbf{T}}(t), \overline{\mathbf{N}}(t), \overline{\mathbf{B}_{\mathbf{1}}}(t), \overline{\mathbf{B}_{\mathbf{2}}}(t)\right\}$. Here we denote $V_{1}=\overline{\mathbf{T}}, V_{2}=\overline{\mathbf{N}}, V_{3}=\overline{\mathbf{B}_{\mathbf{1}}}, V_{4}=\overline{\mathbf{B}_{\mathbf{2}}}$.

Theorem 3.2. Let $\gamma$ be a regular curve in $\mathbb{E}^{4}$ with $\operatorname{PTF}\left\{\overline{\mathbf{T}}(t), \overline{\mathbf{N}}(t), \overline{\mathbf{B}_{1}}(t), \overline{\mathbf{B}_{2}}(t)\right\}$. If the curve $\gamma$ is a $(1,2)$-type slant helix in $\mathbb{E}^{4}$, then we have

and

$$
\left\langle\overline{B_{1}}, \mathbf{U}\right\rangle=\frac{\overline{\kappa_{1}}}{\overline{\kappa_{2}}} c_{1}
$$

$$
\left\langle\overline{B_{2}}, \mathbf{U}\right\rangle=\frac{-\overline{\kappa_{1}}}{\tan \theta \overline{\kappa_{3}}} c_{2},
$$

where $c_{1}$ are $c_{2}$ are constants.

Proof. If the curve $\gamma$ is a $(1,2)$-type slant helix in $\mathbb{E}^{4}$, then for a constant field $\mathbf{U}$, we can write

$$
\langle\bar{T}, \mathbf{U}\rangle=c_{1}
$$

and

$$
\langle\bar{N}, \mathbf{U}\rangle=c_{2}
$$

are constants. Differentiating (3.1) and (3.2) with respect to $t$, we get

$$
\left\langle\bar{T}^{\prime}, \mathbf{U}\right\rangle=0
$$

and

$$
\left\langle\bar{N}^{\prime}, \mathbf{U}\right\rangle=0
$$

Using PTF, we find the following equations:

$$
\left\langle\overline{\kappa_{1}} \bar{N}+\tan \theta \overline{\kappa_{3}} \overline{B_{2}}, \mathbf{U}\right\rangle=0
$$

and it follows that

$$
\left\langle-\overline{\kappa_{1}} \bar{T}+\overline{\kappa_{2}} \overline{B_{1}}, \mathbf{U}\right\rangle=0 .
$$

In that case, we have

$$
\begin{gathered}
\overline{\kappa_{1}}\langle\bar{N}, \mathbf{U}\rangle+\tan \theta \overline{\kappa_{3}}\left\langle\overline{B_{2}}, \mathbf{U}\right\rangle=0 \\
-\overline{\kappa_{1}}\langle\bar{T}, \mathbf{U}\rangle+\overline{\kappa_{2}}\left\langle\overline{B_{1}}, \mathbf{U}\right\rangle=0 .
\end{gathered}
$$

By setting (3.1) in (3.4), we find

$$
-\overline{\kappa_{1}} c_{1}+\overline{\kappa_{2}}\left\langle\overline{B_{1}}, \mathbf{U}\right\rangle=0 .
$$

Substituting (3.4) to (3.3), we obtain as below:

$$
\overline{\kappa_{1}} c_{2}+\tan \theta \overline{\kappa_{3}}\left\langle\overline{B_{2}}, \mathbf{U}\right\rangle=0 .
$$

Finally, we obtain

$$
\left\langle\overline{B_{1}}, \mathbf{U}\right\rangle=\frac{\overline{\kappa_{1}}}{\overline{\kappa_{2}}} c_{1}
$$


and for $\theta(t)=$ constant,$\theta(t) \in\left[-\frac{\pi}{2}, \frac{\pi}{2}\right]$

The proof is completed.

$$
\left\langle\overline{B_{2}}, \mathbf{U}\right\rangle=\frac{-\overline{\kappa_{1}}}{\tan \theta \overline{\kappa_{3}}} c_{2}
$$

Theorem 3.3. Let $\gamma$ be a regular curve in $\mathbb{E}^{4}$ with $\operatorname{PTF}\left\{\overline{\mathbf{T}}(t), \overline{\mathbf{N}}(t), \overline{\mathbf{B}_{1}}(t), \overline{\mathbf{B}_{2}}(t)\right\}$. Then, there are no $(1,3)$-type slant helix in $\mathbb{E}^{4}$.

Proof. If the curve $\gamma$ is a $(1,3)$-type slant helix in $\mathbb{E}^{4}$, then for a constant field $\mathbf{U}$, we can write as below:

$$
\langle\bar{T}, \mathbf{U}\rangle=c_{1}
$$

and

$$
\left\langle\overline{B_{1}}, \mathbf{U}\right\rangle=c_{3}
$$

are constants. Differentiating (3.5) and (3.6) with respect to $t$, we get

$$
\left\langle\bar{T}^{\prime}, \mathbf{U}\right\rangle=0
$$

and

$$
\left\langle{\overline{B_{1}}}^{\prime}, \mathbf{U}\right\rangle=0
$$

Using PTF, we obtain the following equations:

$$
\overline{\kappa_{1}}\langle\bar{N}, \mathbf{U}\rangle+\tan \theta \overline{\kappa_{3}}\left\langle\overline{B_{2}}, \mathbf{U}\right\rangle=0
$$

and we have that

$$
-\overline{\kappa_{2}}\langle\bar{N}, \mathbf{U}\rangle+\overline{\kappa_{3}}\left\langle\overline{B_{2}}, \mathbf{U}\right\rangle=0 .
$$

( We know that $\mathbf{U}$ is constant). By setting equation (3.8) in equation (3.7), we get

it is clear that

$$
\left(\tan \theta \frac{\overline{\kappa_{2}} \overline{\kappa_{3}}}{\overline{\kappa_{1}}}+\overline{\kappa_{3}}\right)\left\langle\overline{B_{2}}, \mathbf{U}\right\rangle=0
$$

$$
\tan \theta \frac{\overline{\kappa_{2}} \overline{\kappa_{3}}}{\overline{\kappa_{1}}}+\overline{\kappa_{3}} \neq 0
$$

Then $\left\langle\overline{B_{2}}, \mathbf{U}\right\rangle=0$, this means that $\mathbf{U}$ is orthogonal to $\overline{B_{2}}$. Then, there are no (1,3)-type slant helices in $\mathbb{E}^{4}$.

Theorem 3.4. Let $\gamma$ be a regular curve in $\mathbb{E}^{4}$ with PTF $\left\{\overline{\mathbf{T}}(t), \overline{\mathbf{N}}(t), \overline{\mathbf{B}_{\mathbf{1}}}(t), \overline{\mathbf{B}_{\mathbf{2}}}(t)\right\}$. If the curve $\gamma$ is a $(1,4)$-type slant helix in $\mathbb{E}^{4}$, then there exists a constant such that

and

$$
\langle\bar{N}, \mathbf{U}\rangle=-\tan \theta \frac{\overline{\kappa_{3}}}{\overline{\kappa_{1}}} c_{4}
$$

$$
\left\langle\overline{B_{1}}, \mathbf{U}\right\rangle=-\tan \theta c_{1}
$$

where $c_{1}$ are $c_{4}$ are constants.

Proof. Assume that $\gamma$ is a $(1,4)$-type slant helix in $\mathbb{E}^{4}$, then for a constant field $\mathbf{U}$, we can write the following equations:

$$
\langle\bar{T}, \mathbf{U}\rangle=c_{1}
$$

and

$$
\left\langle\overline{B_{2}}, \mathbf{U}\right\rangle=c_{4}
$$

are constants. Differentiating (3.9) and (3.10) with respect to $t$, we get

$$
\left\langle\bar{T}^{\prime}, \mathbf{U}\right\rangle=0
$$

and

Using PTF, we get

$$
\left\langle{\overline{B_{2}}}^{\prime}, \mathbf{U}\right\rangle=0 .
$$

$$
\overline{\kappa_{1}}\langle\bar{N}, \mathbf{U}\rangle+\tan \theta \overline{\kappa_{3}}\left\langle\overline{B_{2}}, \mathbf{U}\right\rangle=0
$$


and

$$
\overline{\kappa_{3}}\left\langle\overline{B_{1}}, \mathbf{U}\right\rangle+\tan \theta \overline{\kappa_{3}}\langle\bar{T}, \mathbf{U}\rangle=0
$$

By setting (3.10) in (3.11), we have

$$
\langle\bar{N}, \mathbf{U}\rangle=-\tan \theta \frac{\overline{\kappa_{3}}}{\overline{\kappa_{1}}} c_{4}
$$

Substituting (3.9) to (3.12), we obtain

$$
\left\langle\overline{B_{1}}, \mathbf{U}\right\rangle=-\tan \theta c_{1}
$$

The proof is completed.

Theorem 3.5. Let $\gamma$ be a regular curve in $\mathbb{E}^{4}$ with $\operatorname{PTF}\left\{\overline{\mathbf{T}}(t), \overline{\mathbf{N}}(t), \overline{\mathbf{B}_{\mathbf{1}}}(t), \overline{\mathbf{B}_{\mathbf{2}}}(t)\right\}$. If the curve $\gamma$ is a (2,3)-type slant helix in $\mathbb{E}^{4}$, then there exists a constant such as

$$
\langle\bar{T}, \mathbf{U}\rangle=\frac{\overline{\kappa_{2}}}{\overline{\kappa_{1}}} c_{3}
$$

and

$$
\left\langle\overline{B_{2}}, \mathbf{U}\right\rangle=\frac{\overline{\kappa_{2}}}{\overline{\kappa_{3}}} c_{2}
$$

where $c_{2}$ are $c_{3}$ are constants.

Proof. If the curve $\gamma$ is a $(2,3)$-type slant helix in $\mathbb{E}^{4}$, then for a constant field $\mathbf{U}$, we can write

$$
\langle\bar{N}, \mathbf{U}\rangle=c_{2}
$$

is a constant and

$$
\left\langle\overline{B_{1}}, \mathbf{U}\right\rangle=c_{3}
$$

is a constant. Differentiating (3.13) and (3.14) with respect to $t$, we find

$$
\left\langle\bar{N}^{\prime}, \mathbf{U}\right\rangle=0
$$

and

$$
\left\langle{\overline{B_{1}}}^{\prime}, \mathbf{U}\right\rangle=0 .
$$

Using PTF, the following equations can be obtained:

$$
-\overline{\kappa_{1}}\langle\bar{T}, \mathbf{U}\rangle+\overline{\kappa_{2}}\left\langle\overline{B_{1}}, \mathbf{U}\right\rangle=0
$$

and

$$
-\overline{\kappa_{2}}\langle\bar{N}, \mathbf{U}\rangle+\overline{\kappa_{3}}\left\langle\overline{B_{2}}, \mathbf{U}\right\rangle=0 .
$$

By setting (3.14) in (3.15), we get

$$
\langle\bar{T}, \mathbf{U}\rangle=\frac{\overline{\kappa_{2}}}{\overline{\kappa_{1}}} c_{3}
$$

and substituting (3.13) to (3.16), we have

$$
\left\langle\overline{B_{2}}, \mathbf{U}\right\rangle=\frac{\overline{\kappa_{2}}}{\overline{\kappa_{3}}} c_{2} .
$$

The proof is completed.

Theorem 3.6. Let $\gamma$ be a regular curve in $\mathbb{E}^{4}$ with $\operatorname{PTF}\left\{\overline{\mathbf{T}}(t), \overline{\mathbf{N}}(t), \overline{\mathbf{B}_{\mathbf{1}}}(t), \overline{\mathbf{B}_{\mathbf{2}}}(t)\right\}$. Then, there are no (2,4)-type slant helix in $\mathbb{E}^{4}$. 
Proof. If the curve $\gamma$ is a $(2,4)$-type slant helix in $\mathbb{E}^{4}$, then for a constant field $\mathbf{U}$. We can write the following equations:

$$
\langle\bar{N}, \mathbf{U}\rangle=c_{2}
$$

and

$$
\left\langle\overline{B_{2}}, \mathbf{U}\right\rangle=c_{4}
$$

are constants. By differentiating (3.17) and (3.18) with respect to $t$, we get the following equations:

$$
\left\langle\bar{N}^{\prime}, \mathbf{U}\right\rangle=0
$$

and

Using PTF, we find

$$
\left\langle{\overline{B_{2}}}^{\prime}, \mathbf{U}\right\rangle=0
$$

$$
\begin{gathered}
-\overline{\kappa_{1}}\langle\bar{T}, \mathbf{U}\rangle+\overline{\kappa_{2}}\left\langle\overline{B_{1}}, \mathbf{U}\right\rangle=0, \\
\tan \theta \overline{\kappa_{3}}\langle\bar{T}, \mathbf{U}\rangle+\overline{\kappa_{3}}\left\langle\overline{B_{1}}, \mathbf{U}\right\rangle=0 .
\end{gathered}
$$

Substituting (3.19) to (3.20), we obtain as follows:

$$
\left(\tan \theta \frac{\overline{\kappa_{2}}}{\overline{\kappa_{1}}}+1\right) \overline{\kappa_{3}}\left\langle\overline{B_{1}}, \mathbf{U}\right\rangle=0
$$

it is clear that

$$
\tan \theta \overline{\kappa_{3}} \frac{\overline{\kappa_{2}}}{\overline{\kappa_{1}}}+\overline{\kappa_{3}} \neq 0 \text {. }
$$

Then $\left\langle\overline{B_{1}}, \mathbf{U}\right\rangle=0$, this means that $\mathbf{U}$ is orthogonal to $\overline{B_{1}}$. Then, there are no $(2,4)$-type slant helices in $\mathbb{E}^{4}$.

Theorem 3.7. Let $\gamma$ be a regular curve in $\mathbb{E}^{4}$ with PTF $\left\{\overline{\mathbf{T}}(t), \overline{\mathbf{N}}(t), \overline{\mathbf{B}_{1}}(t), \overline{\mathbf{B}_{2}}(t)\right\}$. If the curve $\gamma$ is a $(3,4)$-type slant helix in $\mathbb{E}^{4}$, then there exists a constant such that

$$
\langle\bar{T}, \mathbf{U}\rangle=-\frac{c_{3}}{\tan \theta}
$$

and

where $c_{3}, c_{4}$ are constants.

$$
\langle\bar{N}, \mathbf{U}\rangle=\frac{\overline{\kappa_{3}}}{\overline{\kappa_{2}}} c_{4}
$$

Proof. If the curve $\gamma$ is a $(3,4)$-type slant helix in $\mathbb{E}^{4}$, then for a constant field $\mathbf{U}$, we can write as follows:

$$
\left\langle\overline{B_{1}}, \mathbf{U}\right\rangle=c_{3}
$$

is a constant and

$$
\left\langle\overline{B_{2}}, \mathbf{U}\right\rangle=c_{4}
$$

is a constant. By differentiating (3.21) and (3.22) with respect to $t$, we have the following equations:

$$
\left\langle{\overline{B_{1}}}^{\prime}, \mathbf{U}\right\rangle=0
$$

and

$$
\left\langle{\overline{B_{2}}}^{\prime}, \mathbf{U}\right\rangle=0
$$

Using PTF, we find as below:

$$
-\tan \theta \overline{\kappa_{3}}\langle\bar{T}, \mathbf{U}\rangle-\overline{\kappa_{3}}\left\langle\overline{B_{1}}, \mathbf{U}\right\rangle=0
$$

and

$$
-\overline{\kappa_{2}}\langle\bar{N}, \mathbf{U}\rangle+\overline{\kappa_{3}}\left\langle\overline{B_{2}}, \mathbf{U}\right\rangle=0 .
$$

Substituting (3.21) to (3.23) and for $\theta(t) \in\left[-\frac{\pi}{2}, \frac{\pi}{2}\right]$ we can write

and by setting (3.22) in (3.24), we obtain

$$
\langle\bar{T}, \mathbf{U}\rangle=-\frac{c_{3}}{\tan \theta},
$$

$$
\langle\bar{N}, \mathbf{U}\rangle=\frac{\overline{\kappa_{3}}}{\overline{\kappa_{2}}} c_{4} .
$$


The proof is completed.

Example 3.8. Let $\gamma(t)=\left(\cos \frac{\sqrt{2} t}{\sqrt{3}}, \sin \frac{\sqrt{2} t}{\sqrt{3}},-\cos \frac{t}{\sqrt{3}},-\sin \frac{t}{\sqrt{3}}\right)$ be a regular unit speed curve in $\mathbb{E}^{4}$ with Frenet frame $\left\{\mathbf{T}(t), \mathbf{N}(t), \mathbf{B}_{1}(t), \mathbf{B}_{2}(t)\right\}$. Frenet frame formulas can be expressed as:

$$
\begin{aligned}
& \mathbf{T}(t)=\left(\frac{1}{\sqrt{3}} \sin \frac{\sqrt{2} t}{\sqrt{3}},-\frac{1}{\sqrt{3}} \cos \frac{\sqrt{2} t}{\sqrt{3}},-\frac{\sqrt{2}}{\sqrt{3}} \sin \frac{\sqrt{2} t}{\sqrt{3}}, \frac{\sqrt{2}}{\sqrt{3}} \cos \frac{\sqrt{2} t}{\sqrt{3}}\right) \\
& \mathbf{N}(t)=\left(-\frac{2}{\sqrt{5}} \cos \frac{\sqrt{2} t}{\sqrt{3}},-\frac{2}{\sqrt{5}} \sin \frac{\sqrt{2} t}{\sqrt{3}}, \frac{1}{\sqrt{5}} \cos \frac{\sqrt{2} t}{\sqrt{3}}, \frac{1}{\sqrt{5}} \sin \frac{\sqrt{2} t}{\sqrt{3}}\right) \\
& \mathbf{B}_{\mathbf{1}}(t)=\left(-\frac{1}{\sqrt{3}} \sin \frac{\sqrt{2} t}{\sqrt{3}}, \frac{1}{\sqrt{3}} \cos \frac{\sqrt{2} t}{\sqrt{3}},-\frac{\sqrt{2}}{\sqrt{3}} \sin \frac{\sqrt{2} t}{\sqrt{3}}, \frac{\sqrt{2}}{\sqrt{3}} \cos \frac{\sqrt{2}}{\sqrt{3}}\right) \\
& \mathbf{B}_{\mathbf{2}}(t)=\left(-\frac{1}{\sqrt{5}} \cos \frac{\sqrt{2} t}{\sqrt{3}},-\frac{1}{\sqrt{5}} \sin \frac{\sqrt{2} t}{\sqrt{3}},-\frac{2}{\sqrt{5}} \cos \frac{\sqrt{2} t}{\sqrt{3}},-\frac{2}{\sqrt{5}} \sin \frac{\ln }{\sqrt{3}}\right),
\end{aligned}
$$

where

$$
\begin{aligned}
& \kappa_{1}=\frac{1}{\sqrt{3}}, \\
& \kappa_{2}=-\frac{\sqrt{2}}{3 \sqrt{5}}, \\
& \kappa_{3}=\frac{\sqrt{2}}{\sqrt{5}} .
\end{aligned}
$$

Equation (2.3) results are written instead of $\left\{\mathbf{T}(t), \mathbf{N}(t), \mathbf{B}_{\mathbf{1}}(t), \mathbf{B}_{\mathbf{2}}(t)\right\}$ for $\theta(t)=\frac{\pi}{3}$, we find $\left\{\overline{\mathbf{T}}(t), \overline{\mathbf{N}}(t), \overline{\mathbf{B}_{1}}(t), \overline{\mathbf{B}_{2}}(t)\right\}$ and their curvature functions expression as follows

$$
\begin{aligned}
& \overline{\kappa_{1}}=\frac{\sqrt{15}+1}{\sqrt{30}} \\
& \overline{\kappa_{2}}(t)=\frac{3 \sqrt{5}-\sqrt{2}}{6 \sqrt{5}}, \\
& \overline{\kappa_{3}}(t)=\frac{1}{\sqrt{10}} .
\end{aligned}
$$

\section{Conclusion}

In this study, we construct $(k, m)$-type slant helices using a PTF equations in $\mathbb{E}^{4}$. $(1, k)$-type for $2 \leq k \leq 4$ slant helices do not exist according to Frenet frame in $\mathbb{E}^{4}$ obtained in [9]. On the contrary, in our paper, we have investigated a PTF in $\mathbb{E}^{4}$ and furthermore, we have showed that according to PTF in 4-dimensional Euclidean space $\mathbb{E}^{4},(k, m)$-type ( $m$ is constant) for $1 \leq k \leq 4, k \neq m$ ( $m$ is constant) slant helices exist and but (1,3)-type, (2,4)-type slant helices do not exist.

\section{AcKNOWLEDGEMENT}

The authors appreciate anonymous referees for their careful corrections to and valuable comments on the paper.

\section{CONFLICTS OF INTEREST}

The authors declare that there are no conflicts of interest regarding the publication of this article.

\section{Authors Contribution Statement}

All authors jointly worked on the results and they have read and agreed to the published version of the manuscript. 


\section{REFERENCES}

[1] Bektaş, M., Yilmaz, M.Y., ( $k, m)$-type Slant helices for partially null and pseudo null curves in Minkowski space $\mathbb{E}_{1}^{4}$, Applied Mathematics and Nonlinear Sciences, Mathematica, 5(1)(2020), 515-520.

[2] Bishop, R.L., There is more than one way to frame a curve, Amer. Math. Monthly, 82(1975), 246-251.

[3] Bulut, F., Bektaş, M., Special helices on equiform differential geometry of spacelike curves in Minkowski space-time, Commun.Fac.Sci.Univ.Ank.Ser. A1 Math. Stat., 69(2)(2020), 51-62.

[4] Gökçelik, F., Gök, İ., Ekmekci, F.N., Yayli, Y., Characterizations of inclined curves according to parallel transport frame in $\mathbb{E}^{4}$ and bishop frame in $\mathbb{E}^{3}$, Konuralp Journal of Mathematics, 7(1)(2019), 16-24.

[5] Hacisalihoglu, H.H., Diferensiyel Geometri, İnönü Üniversitesi Fen Edebiyat Fakultesi Yayınları (In Turkish), 1983.

[6] http://en.wikipedia.org/wiki/Rotation matrix, Euler angles.

[7] Izumiya, S., Takeuchi, N., New special curves and developable surfaces, Turk. J. Math., 28(2004), 153-164.

[8] Soliman, M.A., Abdel-All, N.H., Hussien, R.A., Youssef, T., Evolution of space curves using type-3 Bishop frame, CJMS., 8(1)(2019), $58-73$.

[9] Yılmaz, M.Y., Bektaş, M., Slant helices of $(k, m)$-type in $\mathbb{E}^{4}$, Acta Univ. Sapientiae, Mathematica, 10(2)(2018), 395-401.

[10] Yılmaz, S., Turgut, M., A new version of Bishop frame and an application to spherical images, J. Math. Anal. Appl., 371(2010), 764-776.

[11] Yilmaz, S., Turgut, M., On the characterizations of inclined curves in Minkowski space-time $\mathbb{E}_{1}^{4}$, International Mathematical Forum, 3(16)(2008), 783-792. 